\title{
Quiroz Ávila, Rubén (Introducción y selección) (2019). Ni calco ni copia. Antología de la Filosofía Peruana contemporánea. Lima: Solar.
}

Peruanicemos la filosofía. Ese pudo haber sido también el título de la antología de la filosofía peruana contemporánea que nos presenta Rubén Quiroz Ávila, pues las conclusiones que se extraen de cada uno de los textos que conforman el libro son las de tratar a la filosofía como algo humano y por lo tanto no ajeno a los peruanos ${ }^{1}$. A pesar de que en la actualidad los géneros literarios (y recordemos que la filosofía siempre a echado mano de esto) se han desarrollado con mucha versatilidad, han dejado atrás lo que fue su gran herramienta de difusión y presentación: la antología. La accesibilidad y facilidad con la que ahora muchos escritores y pensadores tienen para publicar hace de la antología una reliquia; no obstante, tiene que ser reinventada para un género del pensamiento humano en permanente desencanto con lo inerte, como es la filosofía, que revive lo que se supone perecido y finiquita lo que parece eterno.

Esa utilidad, difusión y presentación, nunca se pensó como herramienta válida para la difusión y presentación de la filosofía. Muy por el contrario, se apostaba, se apuesta y se seguirá apostando por los libros unitarios, compilados sobre una temática en específico o papers de investigación, sin reconocer que la funcionalidad de la antología puede comenzar desde su más simple elaboración. Es, pues, el primer acierto de Rubén Quiroz Ávila pensar en una antología que difunda y presente lo que nuestros filósofos peruanos han venido desarrollando en los últimos años sin un criterio unificador más que la obviedad: los filósofos peruanos hacen filosofía. Esto nos permite no tener un eje temático homogeneizador, pero sí encontrar tendencias y tópicos, así como voluntades y posiciones particulares sobre qué es filosofía y cómo está entre nosotros, sin correr el riesgo innecesario de definir qué es filosofía en el Perú. Además de ello, la antología nos permite reconocer la heterogeneidad de las indagaciones filosóficas como a su vez su instalación vigorosa en los debates contemporáneos, objetivos que solo una antología, y precisamente una antología como esta, podrían realizar, 
pues "podríamos plantear que mostrar las tensiones conceptuales y las líneas de acción reflexiva de una comunidad intelectual nos dan señales saludables de sus múltiples existencias" (p. 4).

La antología consta de una introducción a cargo del responsable de la selección y de 13 textos, ordenados de manera cronológica en función a la longevidad y juventud del autor: de Miró Quesada a Zenón Depaz. Sin señalar que pudo ser una debilidad de la antología sino que es más bien una posibilidad luego de su lectura atenta, esquematizaremos en tendencias conceptuales a los autores para realizar una mejor explicación, pues, siguiendo a Marx, el método de investigación (en este caso, el método aplicado en la selección de los textos²) debe ser distinto al método de exposición.

\section{La posibilidad y realización de la/una filosofía peruana y latinoamericana:}

Miró Quesada, Salazar Bondy, David Sobrevilla, Juan Abugattás y Augusto Castro.

¿Existe una filosofía en América Latina que sea auténtica y original? ¿Qué criterios tendría que cumplir para que sea así? ¿Es necesaria la pregunta por la autenticidad, es necesaria la dicotomía que se presentó en las primeras líneas del debate entre Zea y Salazar? ¿Qué efecto tiene este debate? Estas preguntas han recorrido el despertar filosófico de nuestros pensadores contemporáneos, cuya trayectoria no es hermética ni mucho menos unilateral. Es horizontal y abierta. Y eso lo demuestra la actualización constante de estas preguntas.

El texto de Francisco Miró Quesada, "Universalismo y latinoamericanismo", es un testimonio que es a su vez historia y balance. A través de ello, podemos conocer el inicio del debate, el punto central y el desenlace de una primera generación que volvió sobre sus premisas para preguntarse por sus conclusiones. La "guerra en dos frentes" que Miró Quesada realizó le permite conocer desde adentro las motivaciones y esencialidades de los universalistas y de los latinoamericanistas, una dicotomía que Juan Abugattás, en "Latinoamérica: el reto de las redefiniciones", la representará en el enfrentamiento del particularismo 
y el universalismo en el campo humano del saber total y su manifestación concreta a través de fenómenos, como los nacionalismos o la pérdida de identidad. Frente a esto, Abugattás plantea estudiar la real condición del hombre latinoamericano para poder "inventar las categorías que abran las puertas a una nueva comprensión de la situación del hombre en América y de sus opciones". Todo esto, poniendo como categoría central a la categoría de "hombre". Esta necesidad de redefinir nuestra posición como latinoamericanos es en términos de David Sobrevilla, tal vez el más y mejor estudioso de nuestro pensamiento filosófico, repensar nuestra tradición. En su texto, "Repensando la tradición nacional", Sobrevilla desarrolla lo que en la actualidad es ya un programa del pensamiento peruano con nuestra tradición (aquí repensar es reconocer, actualizar y cuestionar a los nuestros) que, no obstante, debe continuar siguiendo hasta llegar a un programa filosófico general (repensar, en este caso, es apropiación y cuestionamiento de la tradición universal), rechazando el anatopismo. Esta tarea tiene que reconstruir los pensamientos de nuestros filósofos peruanos, repensándolos, comprendiendo que "buena o mala, nuestra tradición nos constituye y que, como el pensamiento siempre tiene que partir de su realidad -si no quiere transformarse en fantasía o deseo-, debe partir de esta, actualizarla y cuestionarla en lo que tiene de deficiente" (p. 71).

Augusto Castro por su parte, en "La filosofía entre nosotros. Cinco siglos de Filosofía en el Perú", inicia con una premisa que se cuestiona pero que para él es un hecho: la filosofía existe hace mucho tiempo en nuestras tierras y "nuestros filósofos han pensado las cosas tomando nota principalmente de los problemas que tenían al frente y no tanto de los debates que se daban en el mundo". Pero esto ha sido con deficiencias y para vencer a esos obstáculos Castro propondrá integrar la mirada universal y la mirada propia, pues "pensar sobre el pensamiento y las razones de las cosas es hacer filosofía. Es una manera de dejar la mirada estrecha, sin perder el sentido real" (p. 220). Augusto Salazar Bondy, para quien según Castro no había un duda sobre la existencia de la filosofía sino que no haya una filosofía emancipadora, en el "Comienzo del filosofar" demuestra que, efectivamente, la filosofía ya estaba con nosotros aún incluso cuando el cuestionamiento de su originalidad yacía sobre ella, pues a lo largo de aquel texto 
desarrolla un pensamiento que ayuda a encontrarnos con la filosofía de manera sincera, encaminada hacia un trabajo original: no hay pensamiento nuevo sin un pensamiento que lo antecede y he ahí la importancia de la iniciación, pues iniciarse en "la filosofía significa [...] entrar en el diálogo de los filósofos, aprender su lenguaje, recibir el impacto de sus inquietudes y ser promovido de este modo a un nuevo pensar". Tal diálogo filosófico tiene a su vez una condición necesaria: comprender, a su vez, que la filosofía es meditación personal. Así, para Salazar Bondy ingresamos a la filosofía en la iniciación comprometidos, inevitablemente, en persona "con la reflexión nacional", siendo que, "tradición y originalidad son, en suma, categorías fundamentales del quehacer filosófico. Por eso, aprender filosofía no es repetir una filosofía existente sino llegar, por mediación de un filosofar existente, a un nuevo pensar" (p. 54). Los resultados de esta tendencia conceptual de nuestra filosofía no es meramente un proceso histórico. Es, como ya lo han dicho de Miró Quesada a Castro, reconocer a nuestra filosofía como un hecho.

Intervención en lo real mediante el pensamiento: José Carlos Ballón y Teresa Arrieta.

Sin negar el carácter efectivo de toda filosofía sobre la realidad, los textos de José Carlos Ballón y Teresa Arrieta de Guzmán son más explícitos en su vinculación con una intervención mediata e inmediata sobre el devenir de nuestra sociedad: la corrupción y la contaminación ambiental, respectivamente, son los temas que abordarán con un manejo adecuado y profundo (sobre todo en la segunda) de las categorías filosóficas necesarias.

Procesando a la modernidad, en el sentido de proceso mariateguista, Ballón diagnóstica en "Ética, modernidad y autoritarismo en el Perú actual: ¿vigilar o castigar?", el desarrollo de la modernidad y las aporías que, en su inmanencia, se desenvuelven con ella y cómo la sociedad peruana se opone a la moderna ética del trabajo con su pre-modernidad. Ésta conflictuada relación impide ver a la modernidad como una totalidad y por lo tanto a su totalidad ética. De ahí la corrupción imperante en nuestro país: el apego a lo tradicional, es decir, 
a los hábitos no modernos de vivir de otros y no de un trabajo propio. Por su lado, el texto de Teresa Arrieta de Guzmán, "Ética ambiental: del dominio al respeto a la naturaleza", que es el más extenso de la antología, no solo es una muestra de solidez argumentativa y destreza filosófica sino que también ello le permite realizar mejores críticas a los blancos que la filosofía viene enjuiciando por ser culpables de la crisis de nuestra civilización. Así, criticando las teorías que explican la génesis de la contaminación ambiental (vinculados a la crítica del capitalismo, patriarcado, avance tecnológico, hiperconsumo, etc.), Arrieta de Guzmán supera el simple señalamiento para pasar a realizar una exhaustiva búsqueda no de una causa sino del producto de la suma de esas causas, analizando dicho resultado desde la axiología, el antropocentrismo y el humanismo. Procesa (de nuevo, en su acepción mariateguista) las posturas ético ambientalistas que, reevaluando sus puntos menos débiles, pueden resultar en una suma, que al igual que la suma de causas, puede ser suma de posibilidades hacia la reconstrucción total de una ética. Por el momento, dice Arrieta de Guzmán, "hay urgencia en concientizar a las mayorías en principios que, efectivamente, protejan al ambiente" (p. 197).

José Carlos Ballón propone una búsqueda coherente, tanto crítica como asimiladora, de la modernidad en un camino hacia adelante que busque eliminar la corrupción, que es producto de una ética tradicional y por lo tanto, de un sustento incoherente en el pensamiento del desenvolvimiento práctico. Por su parte, Teresa Arrieta de Guzmán, sin frenar el avance de las indagaciones, propone la necesaria inmediatez de formar hombres y mujeres en base a los principios de la bioética, como primer paso en el combate contra la destrucción de la naturaleza. En ambos la filosofía tiene que realizarse superándose a sí misma: la reinvención de sus premisas rebasando sus contradicciones.

La identidad, lo racional, el otro y la interculturalidad: José Ignacio López Soria, María Luisa Rivara, Edgar Montiel y Pablo Quintanilla.

Junto al texto de Teresa Arrieta de Guzmán, reseñado en el apartado anterior, los textos de María Luisa Rivara y Pablo Quintanilla, "En torno a la identidad nacional" e "Interpretando al otro: comunicación, racionalidad y relativismo", 
respectivamente, demuestran un dominio más explícito, en esta antología, de los mecanismos del filosofar. Aunque naciendo de contradicciones en los objetos y campo de estudio de las Ciencias Sociales, López Soria nos presenta su propuesta de filosofía de la plenitud en "El principio interculturalidad y la filosofía de la plenitud". Más histórico, pero no menos filosófico, Edgar Montiel vuelve a presentarnos en "El Inca Garcilaso y la independencia de las Américas" una relectura de nuestro pasado para ubicar su presencia en los procesos que nos legaron nuestro presente. Todos, de López Soria a Pablo Quintanilla buscan identificar al sujeto con una identidad comunitaria y con acciones colectivas, sin que esto signifique una verdad de perogrullo para convivir en este mundo precario.

Edgar Montiel centra su punto de partida en los "Comentarios reales de los Incas", cuyo espacio y tiempo histórico se remontan más allá de los límites de una literatura, pues lo que rastreará Montiel es la influencia de este texto en las rebeliones de Túpac Amaru y de los precursores de la Independencia. Su permanente influjo en nuestra historia subversiva contra el dominio español se debe al haber insertado en el imaginario intelectual el derecho natural de nuestros pueblos a exigir lo suyo. Pero ¿qué significa nuestro pueblo y qué identidad tiene nuestra nación? María Luisa Rivara responde en su texto que "la búsqueda de una identidad real es, a la vez, pensamiento del ser de esa entidad" (p. 103). En ese sentido, desarrolla su indagación en un diálogo entre el ser y la entidad para comprender la identidad. Rechazará la relación de la lo real con lo idéntico de manera mecánica, pues siendo así se anula la posibilidad de una identidad múltiple que es, en sus conclusiones, la verdadera identidad nacional de nuestra patria. A diferencia de Theodor Adorno, que piensa que lo esencial del ser es lo no idéntico, es decir, lo que no cabe en una conceptualización, lo rechazado, Rivara acepta que lo real siendo idéntico significa que en nuestra identidad cabe lo que es nuestro ser: pluricultural, pluriétnica y plurilingüe. El ejercicio filosófico de Rivara está en no reducir lo real a lo idéntico sino que lo real es la identidad y en base al reconocimiento de nuestra realidad, hallaremos lo que somos. 
López Soria y Quintanilla, por tu parte, establecen un puente entre lo que somos y lo tenemos que ser en nuestra relación con el otro. El primero, a través de la interculturalidad y el segundo, mediado por lo que sería una manera adecuada de comprender la racionalidad, las objeciones relativistas y la construcción de estos dos elementos en uno solo: la comunicación. En ambos autores la relación con el otro no es posible sin el diálogo y la comunicación. No puede haber interculturalidad sin diálogo, señala López Soria. A esto, le suma la tarea de desmontar la violencia que nuestras tradiciones nos han impreso en nuestra cultura. Es pertinente por lo tanto, desbaratar la idea de progreso y desarrollo, sinónimos de la modernidad, que no solamente son un tipo de violencia material sino que también son una violencia epistémica. Pablo Quintanilla por su lado, en un texto donde la lectura tiene que ser exhaustiva por el dominio que hace gala el autor de la filosofía de Donald Davidson, es en recurrido de redefiniciones propias del autor, con el objetivo de entender que la racionalidad es la "posibilidad de crear un espacio de interpretación intencional común, lo que dará lugar a una forma de vida compartida o, para ponerlo en el lenguaje de la hermenéutica gadameriana, a una fusión de horizontes" (p. 236).

Los autores de esta tendencia y/o tendencias filosóficas no solo demuestran propuestas valiosas que aportan al ejercicio del filosofar peruano sino que sobre todo proponen, con actualidad, las categorías harto estudiadas por la filosofía universal.

\section{Hermenéutica del pensamiento andino: Víctor Mazzi y Zenón Depaz.}

El cierre de la antología tiene actualidad no solo en la juventud intelectual de sus autores sino en la indagación y sobre todo en el esclarecimiento del quechua, que desde comienzos de este siglo ha recobrado primacía en las páginas más pujantes y polémicas de nuestro pensamiento y que son publicadas en artículos, libros, columnas de opinión o tesis universitarias. Ambos autores, Mazzi y Depaz, no solo muestran sino que también demuestran, sobre todo el segundo, que en la realización del quechua como idioma se encuentra un lenguaje que para ese entonces y para hoy no solo nombra sino que también representa el ideario de una civilización y su compromiso, siendo consigo mismos, con el mundo. 
Víctor Mazzi presenta en su artículo, "Kay, pacha, yachay. La reflexión y el saber en el Tawantinsuyo", 4 palabras que en el Tahuantinsuyo eran usadas para designar, en su formación original, un sentido, una realidad y un comprender, pero que siendo aglutinante el quechua y a través de sufijos, estas palabras designaban mayores sentidos, realidades y comprensiones que el uno solo original. Sin fungir de diccionario, Mazzi logra darnos un texto cuya importancia radica en ubicar, a los interesados en la especialización o a los curiosos del pensamiento andino, la relación del quechua y el horizonte que designa al hacerse diálogo y voz. Cumpliendo otros objetivos, pero bajo las mismas premisas, Depaz en "Experiencia cósmica y dinámica social en el Manuscrito de Huarochirí" realiza su investigación motivado por lo que siendo horizonte en el mundo andino es hoy condición de posibilidad para "la construcción de la patria grande latinoamericana" (p. 308). Al igual que Mazzi, en el texto de Depaz hay una explicación de cómo el idioma designa, pero además, y es lo que distingue uno de otro texto, cómo relaciona y de esta manera, como fue la relación en el mundo andino, ubicando el autor lo que ha de ser el aporte de la cosmovisión andina: el hombre no es en el mundo sino que es con el mundo. De esta manera, el cierre de la antología es una invitación a indagar sobre nuestro pensamiento andino como ejemplo de posibles y futuras indagaciones de los diversos pensamientos milenarios que perviven en nuestro país.

\section{Conclusiones}

La importancia de la publicación de esta antología tiene un carácter urgente en su difusión y promoción, pues para esta ha nacido y de esta pueden devenir muchos primeros contactos de los interesados en la filosofía y el filosofar de nuestro pensamiento racional, totalizador, sistemático y radical y crítica. No obstante sus aciertos, sus desaciertos vendrían en su forma: la necesidad de un índice titulado con los nombres de los artículos y una bibliografía de cada uno de los autores, siendo de esta manera una condición ineludible explicar con un poco más de detalle en qué proceso del filosofar particular de cada autor se inserta el texto antologado. 
Nuestra filosofía se tiene que peruanizar mostrándola, difundiéndola. Las producciones académicas, hoy muy en boga en las universidades peruanas, solo podrán ser nuestras cuando bajen al llano y el método más adecuado no es reduciendo su rigurosidad o impostar una sencillez inoportuna sino más bien a través de formatos harto conocidos y masivos, cuyo paso previo, la investigación para la antología, no tiene que tener más objetivos, debido a su urgencia, que el de mostrar. Urge vernos y reconocernos en la palabra filosofía y en el ejercicio del filosofar. Esta antología es un encuentro y hacia el encuentro de nuestra filosofía debemos partir. No demoremos en el encuentro con los nuestros dado que siendo ellos, somos nosotros mismos.

\section{Notas}

1 Recordemos a Mariátegui y las líneas que iza como bandera en el primer número de Amauta: "Todo lo humano es nuestro".

2 La antología como método de investigación es una idea que habría que indagar más en nuestro pensar filosófico y como ésta, olvidada en la literatura, puede ser de utilidad para la difusión y presentación de nuestros filósofos no solo contemporáneos sino de antaño, sin otro patrón más que el de ubicar lo más esencial de su pensamiento y presentarlo como son: pensamientos que indagan.

\section{Miguel Ángel Malpica}

Universidad Nacional Mayor de San Marcos, Lima, Perú.

Contacto: miguiel.malpica1@unmsm.edu.pe

https://orcid.org/0000-0001-7797-1321 